\title{
Tooth Fragment Reattachment: An Esthetic, Biological Restoration
}

\author{
Nitin Kararia, Ajay Chaudhary, Vandana Kararia
}

\begin{abstract}
Coronal fractures of the anterior teeth are a common form of dental trauma. If the original tooth fragment is retained following fracture, reattachment of the fractured fragment to the remaining tooth can provide better and long-lasting esthetics, improved function, a positive psychological response and is a faster and less complicated procedure. This paper reports on two coronal tooth fracture cases that were successfully treated using adhesive reattachment technique.
\end{abstract}

Keywords: Coronal fracture, Dental trauma, Reattachment, Esthetics, Resin composite.

How to cite this article: Kararia N, Chaudhary A, Kararia V. Tooth Fragment Reattachment: An Esthetic, Biological Restoration. World J Dent 2012;3(1):91-94.

Source of support: Nil

Conflict of interest: None declared

\section{INTRODUCTION}

Coronal fractures of the anterior teeth are a common form of dental trauma that mainly affects the maxillary incisors because of their position in the arch. ${ }^{1,2}$ Factors that influence the management of coronal tooth fractures include the site of fracture, size of fractured fragments, periodontal status, pulpal involvement, root maturation, biological width invasion, occlusion and time.

One of the options for managing coronal tooth fractures, especially when there is minimal or no violation of the biological width, and the fractured fragment is retained, is the reattachment of the dental fragment. Reattachment of a fragment to the fractured tooth can provide good and long-lasting esthetics because the tooth's original anatomic form, color and surface texture are maintained. Additionally, it generates a positive psychological response and is a reasonably simple procedure that provides a more predictable long-term wear than when a direct composite is used. $^{3}$

The concept of reattachment began in 1964 when Chosak and Eidelman used a cast post and conventional cement to reattach an anterior crown segment. ${ }^{4}$ Recent developments in restorative materials, placement techniques and adhesive protocols allow reattachment using resin based composites. Tannery was the first to use acid etch technique for the reattachment of fractured tooth fragment..$^{5}$ Subsequently, Starkey and Simonson have reported similar cases. ${ }^{6,7}$
This article reports on two coronal tooth fracture cases that were successfully treated using tooth fragment reattachment.

\section{CASE REPORTS}

\section{Case 1}

A 22-year-old female patient reported to the Department of Conservative Dentistry and Endodontics at Rajasthan Dental College with the chief complaint of fractured upper anterior teeth due to a road traffic accident, a day before. Patient's medical history was noncontributory. Clinical and radiographic examination revealed horizontal fractures (Ellis class III) in the middle-third regions of the crowns of maxillary right central incisor and left lateral incisor (Figs 1 and 2). Patient was in acute pain due to pulp exposure with both the teeth. No mobility of the injured teeth was recorded and surrounding intraoral soft tissues were normal. Extraoral examination revealed a wound on the left side of her upper lip (Fig. 3). On palpation the lip felt hard in that area. The mouth of the wound was carefully explored with a sterile probe which yielded the fractured coronal fragment of a tooth (Fig. 4). On checking for adaptation, the fractured fragment fitted well on the maxillary left lateral incisor, confirming that it was the fractured incisal segment of the same tooth that created a penetrating wound in the patient's upper lip and was lodged there. The fragment was washed thoroughly under running water and stored in sterile normal saline to prevent dehydration and discoloration. The patient

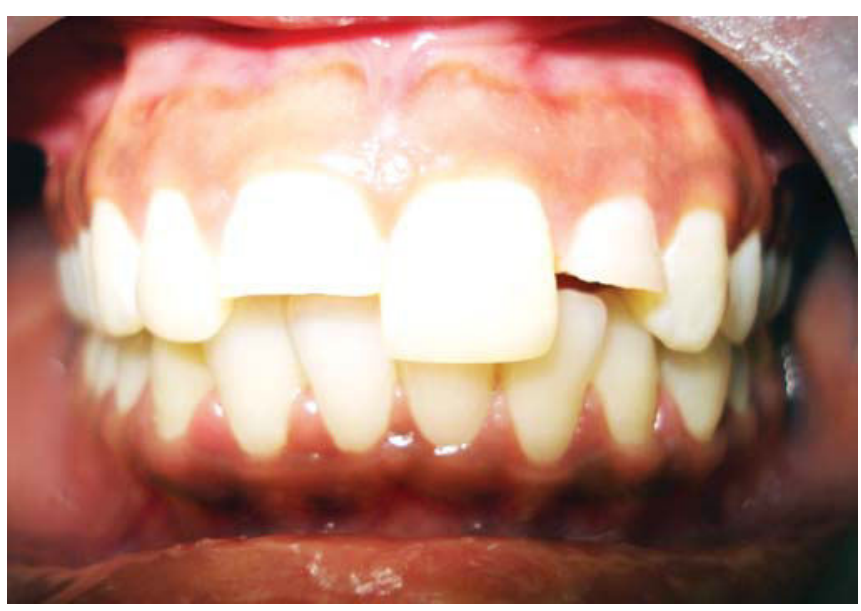

Fig. 1: Preoperative frontal view 


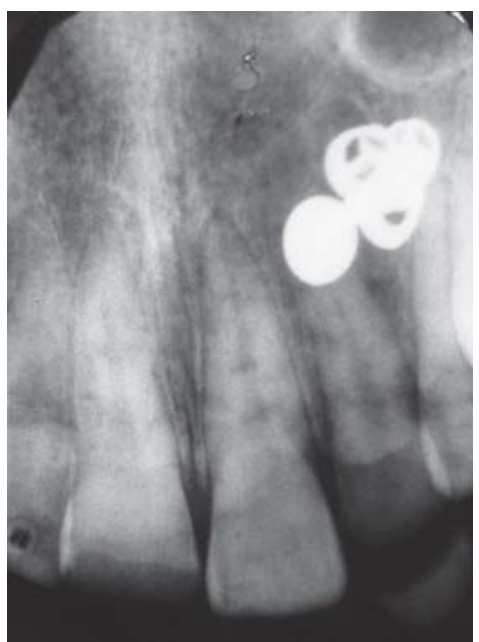

Fig. 2: Preoperative radiograph

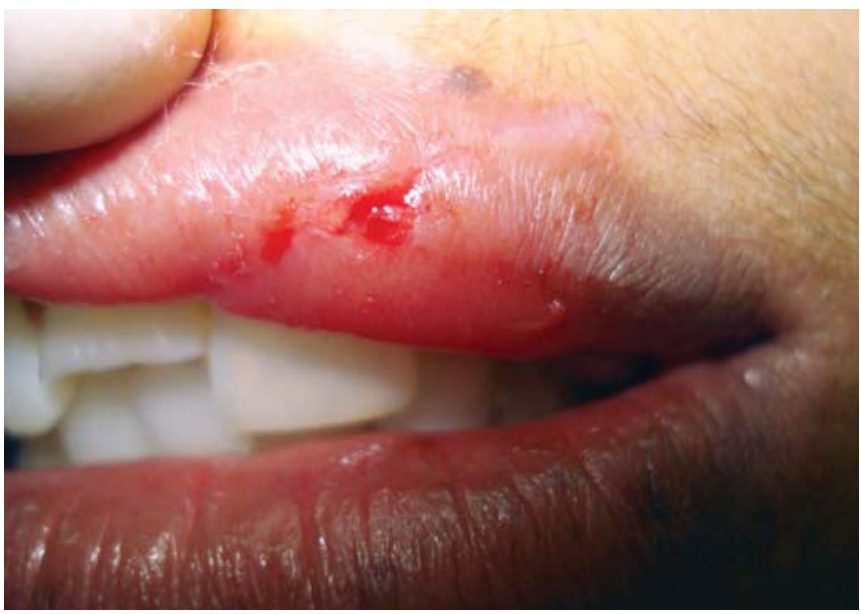

Fig. 3: Upper lip with lacerated wound

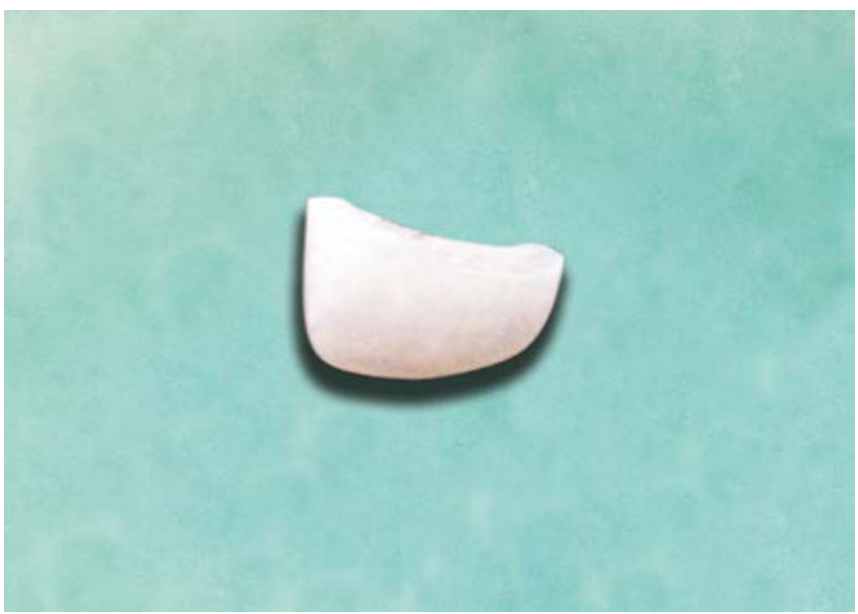

Fig. 4: Fractured coronal tooth fragment

expressed the desire to maintain teeth and restore them. It was planned to reattach the fractured fragment to the remaining tooth and patient's consent was obtained. Root canal treatment was carried out with both the fractured incisors. In the left lateral incisor the gutta-percha points

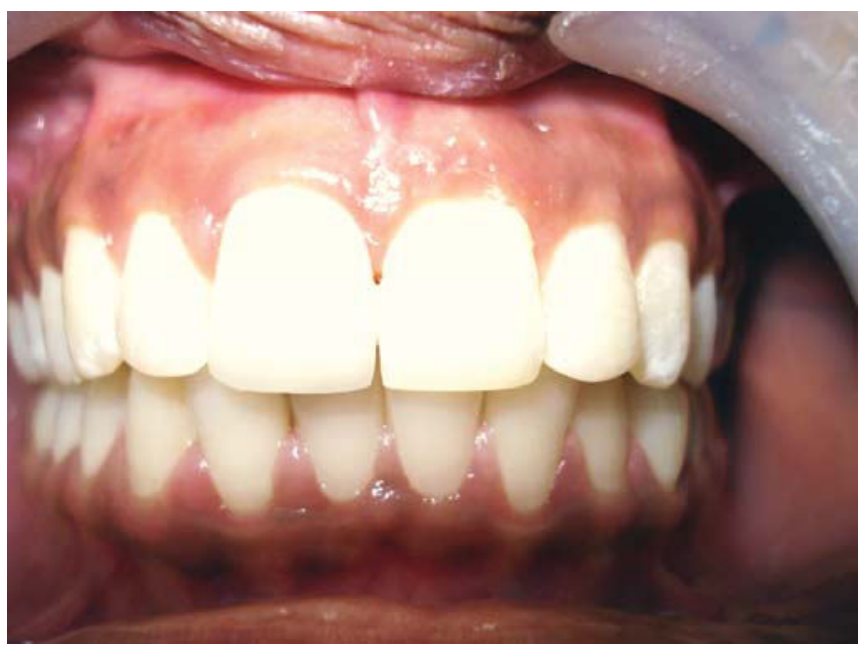

Fig. 5: Postoperative frontal view

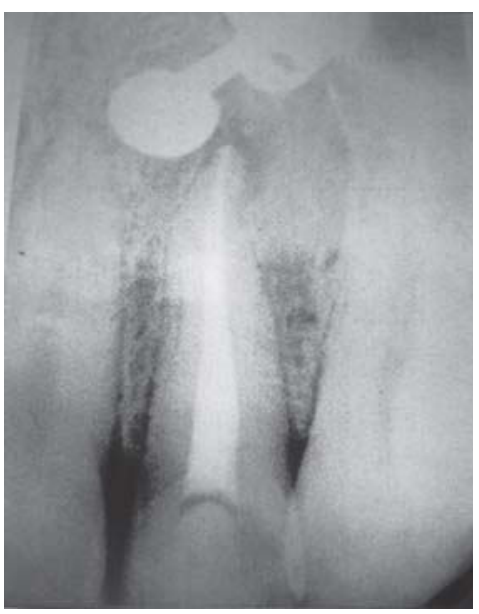

Fig. 6: Postoperative radiograph

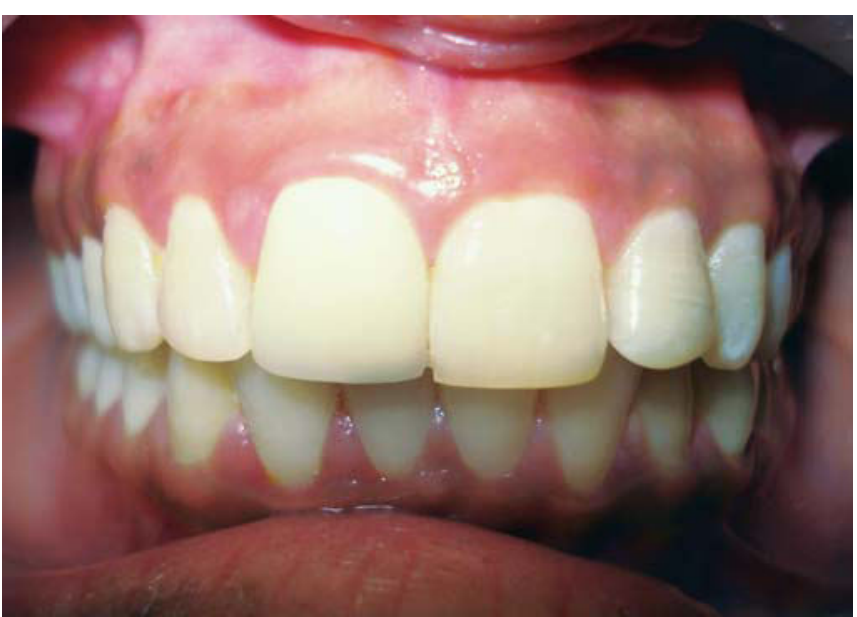

Fig. 7: Frontal view (4 months follow-up)

were removed to the level of cement enamel junction and the canal orifice was sealed with glass ionomer cement. The tooth's fractured surface was then etched with $37 \%$ orthophosphoric acid, rinsed, blot dried with paper points and bonding agent (Prime and Bond NT, Dentsply) was 


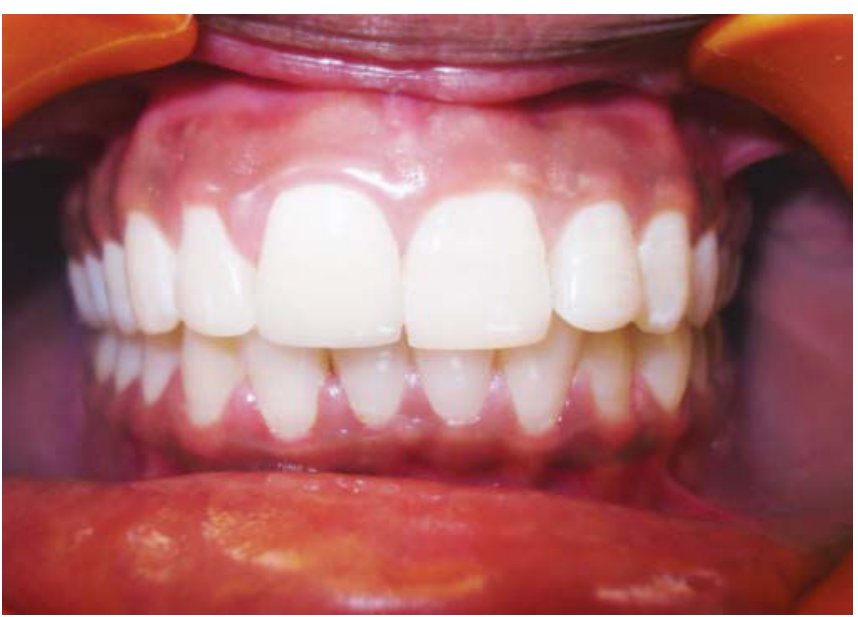

Fig. 8: Frontal view (12 months follow-up)

applied. Small grooves were prepared in the inner portion of fractured coronal fragment and its inside was etched, rinsed and bonded. Subsequently, flowable composite (Grandio Flow, Voco) was used to fill the access cavity in the tooth and the prepared grooves into the coronal fragment. The fragment was carefully seated on the remaining tooth and light cured. During curing firm and stable finger pressure was applied to the coronal fragment to closely appose it to the tooth. After curing, excess composite was removed with a diamond finishing bur. Afterward final polishing was done with Enhance (Dentsply) (Figs 5 and 6). Follow-up examinations were carried out at 4 months and 1 year intervals, during which tooth remained normal in esthetics and function (Figs 7 and 8). Maxillary right central incisor was restored with post and core followed by metal ceramic crown.

\section{Case 2}

A 32-year-old female reported with uncomplicated crown fracture in the middle-third of maxillary left lateral incisor due to a fall in the bathroom (Fig. 9). The fractured tooth fragment was recovered from her and stored in sterile normal saline (Fig. 10). Upon examination the treatment plan of choice was to reattach the coronal fragment to the remaining tooth. Patient's consent was obtained and endodontic therapy was carried out in the remaining tooth. Subsequently, the coronal tooth fragment was reattached to the remaining tooth using the same procedure like that followed in case 1 (Figs 11 and 12). Follow-up examinations were carried out at 20 months interval (Fig. 13). The tooth remained normal in esthetics and function.

\section{DISCUSSION}

The remarkable advancement of adhesive systems and resin composites has made reattachment of tooth fragments a procedure that is no longer a provisional restoration, but rather a restorative treatment offering a favorable prognosis.

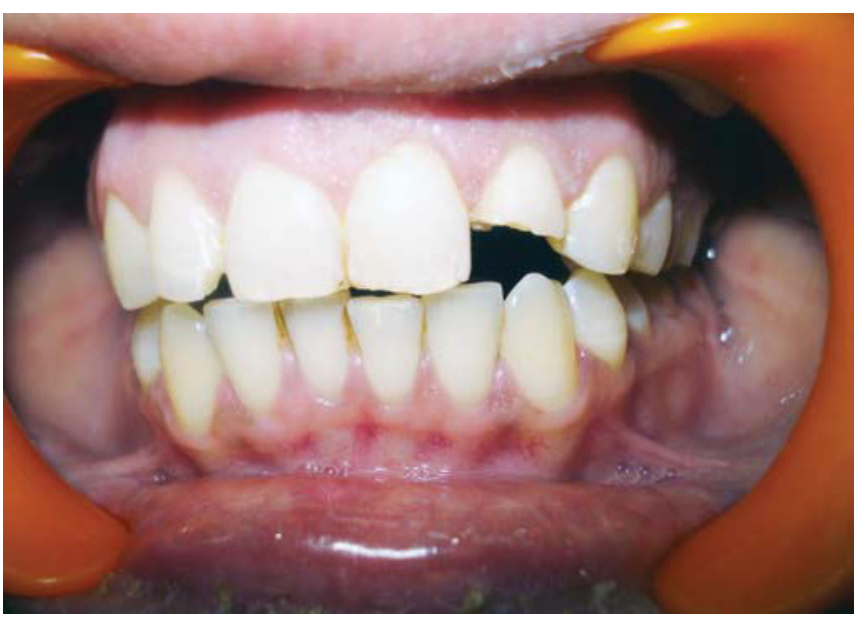

Fig. 9: Preoperative frontal view

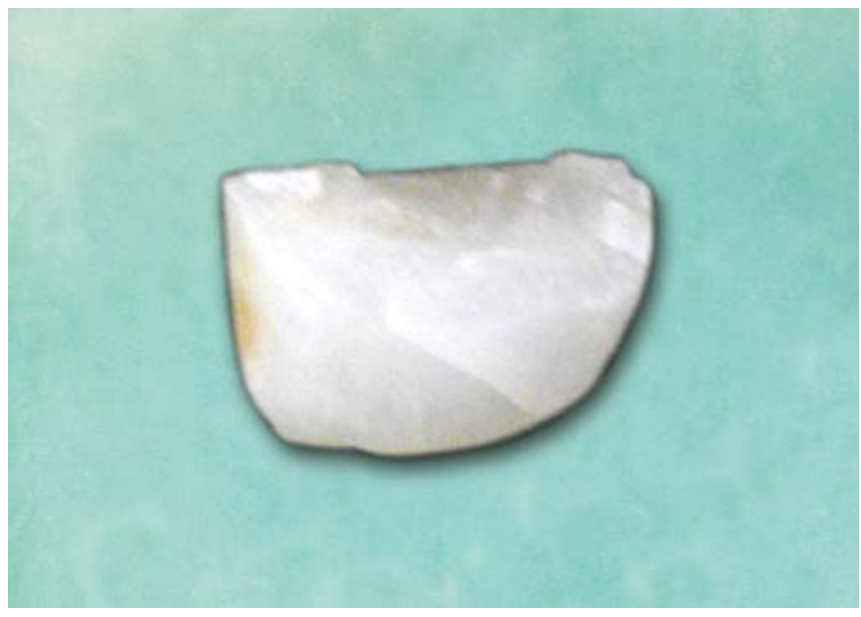

Fig. 10: Fractured tooth fragment-facial view

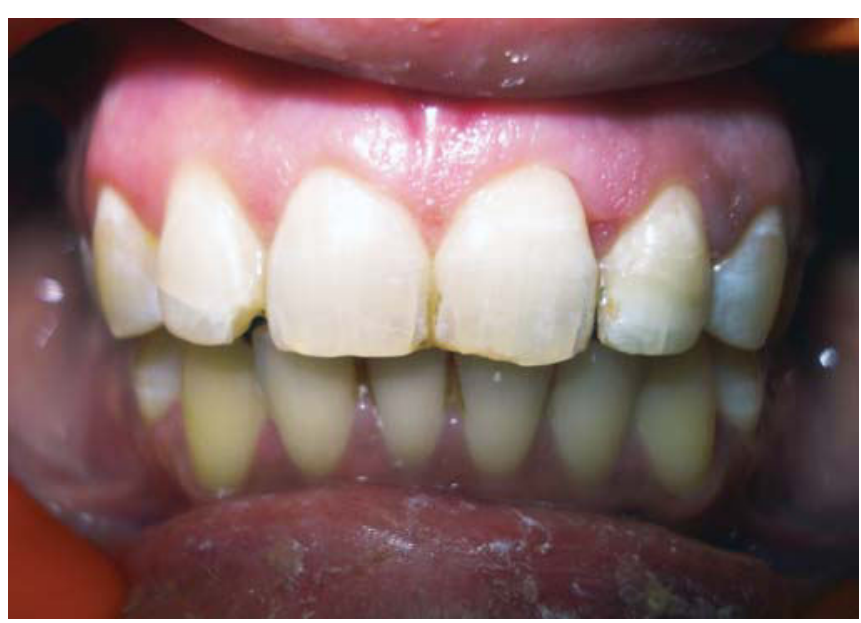

Fig. 11: Postoperative frontal view

However, this technique can be used only when the intact tooth fragment is available. ${ }^{8}$

In the present cases, an ultraconservative approach was adopted and the fractured fragments were reattached without the use of post for retention, unlike many previously reported cases. ${ }^{9,10}$ In both the cases excellent long-term results were 


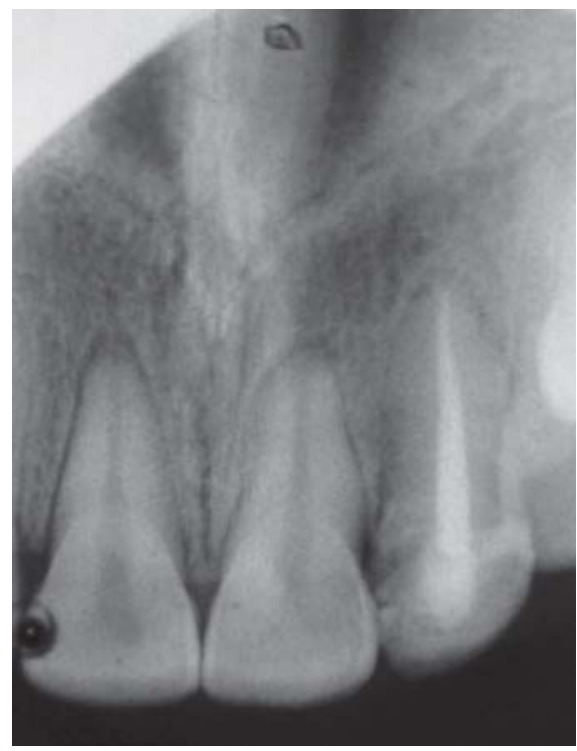

Fig. 12: Postoperative radiograph

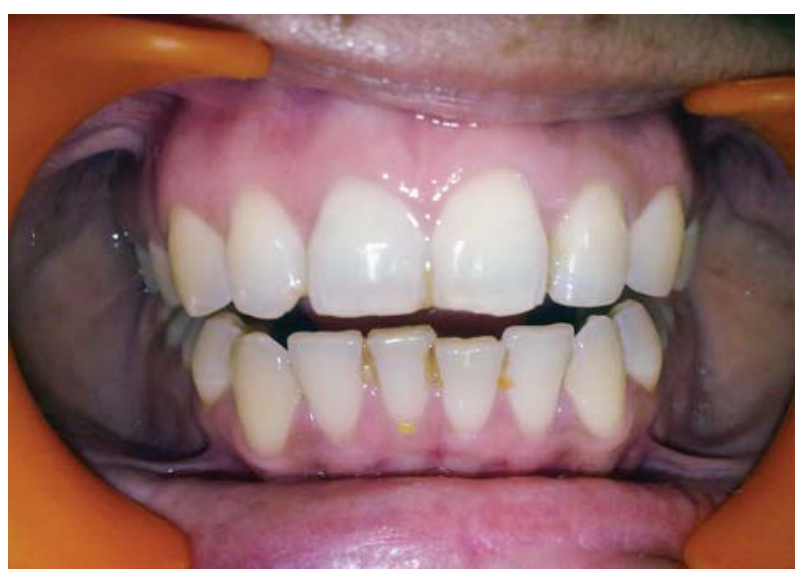

Fig. 13: Frontal view (20 months follow-up)

obtained. Thus, it could be concluded that tooth fragment reattachment is a viable technique that restores function and esthetics with a very conservative approach, and should be considered when treating patients with coronal fractures of the anterior teeth.

\section{REFERENCES}

1. Dietschi D, Jacoby T, Dietschi JM, Schatz JP. Treatment of traumatic injuries in the front teeth: Restorative aspects in crown fractures. Pract Periodontic Aesthet Dent 2000;12(8):751-58.
2. Hamilton FA, Baratieri LN, Hill FJ, Holloway PJ. An investigation of dentoalveolar trauma and its treatment in an adolescent population. Part 1: The prevalence and incidence of injuries and the extent and adequacy of treatment received. $\mathrm{Br}$ Dent J 1997;182:91-95.

3. Monteiro S Jr, Andrada MAC. Tooth fracture reattachment: Case reports. Quintessence Int 1990;21(4):261-70.

4. Chosak A, Eidelman E. Rehabilitation of a fractured incisor using patient's natural crown: Case report. J Dent Child 1964;31: 19-21.

5. Tennery NT. The fractured tooth reunited using the acid etch bonding technique. Tex Dent J 1988;96:16-17.

6. Starkey PE. Reattachment of a fractured fragment to a tooth. J Ind Dent Assoc 1979;58:37-38.

7. Simonsen RJ, Osborne JW, Lamsen RL. Restoration of a fractured central incisor using original teeth. J Am Dent Assoc 1982;105:646-48.

8. Simonsen RJ. Traumatic fracture restorations: An alternative use of the acid etch technique. Quint Int 1979;10(2):15-22.

9. Christensen GJ. When to use fillers, build-ups or posts and cores. J Am Dent Assoc 1996;127:1397-98.

10. Hayashi M, Takahashi Y, Imazato S, Ebisu S. Fracture resistance of pulpless teeth restored with post-cores and crowns. Dent Mater 2006;22:477-85.

\section{ABOUT THE AUTHORS}

\section{Nitin Kararia}

Senior Lecturer, Department of Conservative Dentistry and Endodontics, Rajasthan Dental College and Hospital, Jaipur Rajasthan, India

\section{Ajay Chaudhary}

Professor, Department of Conservative Dentistry and Endodontics Rajasthan Dental College and Hospital, Jaipur, Rajasthan, India

\section{Vandana Kararia}

Senior Demonstrator, Department of Orthodontics, Government Dental College and Hospital, Jaipur, Rajasthan, India

\section{CORRESPONDING AUTHOR}

Nitin Kararia, Senior Lecturer, Department of Conservative Dentistry and Endodontics, 138, Sahyog II Apartments, Sector 6, Vidhyadhar Nagar, Jaipur-302023, Rajasthan, India, Phone: 91-9829114149 e-mail: amazingsaggian@yahoo.com 